\title{
Prototype Design of Indigenous GSM based Intelligent Irrigation System
}

\author{
Ripul Rishi \\ ACSD Deptt. \\ C-DAC, Mohali
}

\author{
Dilip Kumar \\ ACSD Deptt. \\ C-DAC, Mohali
}

\begin{abstract}
The majorities of soil moisture sensor being used in India for the detection of the moisture content of the field is imported and are very costly. There is a need to design new indigenous GSM based intelligent irrigation system. The key intent of this paper is to testimony an indigenous GSM based intelligent irrigation system which performs gathering of moisture from the field with the help of developed sensor and derive suitable actuator(i:e motor). The moisture can also be known from a remote place using handheld device.
\end{abstract}

\section{Keywords}

GSM, Intelligent irrigation, Microcontroller.

\section{INTRODUCTION}

Recent research focus on moisture measurement, control irrigation, continuous and remote monitoring for better crop growth and resourceful water management. In (F.Adama; G.Andria; F.Attivissimo; L.Fabbianno; N.Giapuinto, 2007), the design and the development of innovative soil moisture sensor for agriculture application is presented. The development of the sensor not only solves the moisture problem, therefore there is a need of developing the intelligent system for continuous and remote monitoring with irrigation control.

The moisture measurement is essential these days for agriculture applications because different crops need different moisture level and that level need to be maintained for the better growth of crop. To solve the inconvenience of real time techniques a remote environmental monitoring system was designed (Zhuanwei Wang; Chunjiang Zhao; Haihui Zhang; Hangpan Fan, 2011).

The presented paper deals with the design of indigenous gsm based intelligent irrigation system using AT89S52 microcontroller. As shown in Figure1 the controller circuit consists of microcontroller unit consisting of AT89S52 microcontroller (CPU), some peripherals like liquid crystal display (LCD), 2-key keypad, analog to digital convertor (ADC0808), pump, handheld device, GSM (sim300) and moisture sensor.

\section{SYSTEMIC DESCRIPTION}

The whole on circuit is based on microcontroller with moisture sensor connected to and a gsm module controlled by microcontroller. All the circuit components were selected to provide to provide the desired functionality and they are easily available in the market. The system is suitable for all kinds of agriculture soils. The indigenous moisture sensor developed is used to get the moisture from the field.

\subsection{Circuit intend}

The main components of the circuit intended are programmable microcontroller, GSM (sim300), voltage regulator, relay, LCD.

\subsubsection{Power supply}

The microcontroller and the peripherals including keypad, lcd, relay, $\operatorname{gsm}(\operatorname{sim} 300)$, uses a $12 \mathrm{VDC}$ adaptor. The LM7805 will step down $12 \mathrm{VDC}$ to $5 \mathrm{VDC}$ output to microcontroller, ADC and LCD.

\subsubsection{Microcontroller}

The microcontroller used is AT89S52(Atmel), low power, high performance CMOS 8-bit, 32 programmable input output lines, 256x8-bit internal ram, eight interrupt sources. It is a powerful microcontroller which provides highly flexible and cost effective solution to many embedded control applications. It has three 16-bit timer/counter. The clock frequency is $11.0592 \mathrm{MHz}$

\subsubsection{LCD}

A $16 \times 2$ LCD is connected to port1 of microcontroller. It display received signal from microcontroller.

\subsubsection{Keypad}

A 1x1 keypad is connected to microcontroller for the desired inputs of the moisture according to the crop grown in the field. It is connected to port 3 of microcontroller.

\subsubsection{GSM}

The GSM module is used for remote monitoring. It is used to send and receive the message. The default band used is EGSM 900 and DCS 1800. It supports protocol PAP (Password Authentication Protocol) usually used for ppp connection. It has a real time clock implemented. It has two serial interfaces serial port 1 can be used for GPRS service and send AT command of controlling mode. Serial port 2 has two lines on serial port interface i:e TXD and RXD. Serial port2 is only used for transmitting AT commands. It supports transmission of message alternatively over CSD or GPRS. Preferred mode can be selected. Baud rate used is 9600 . 


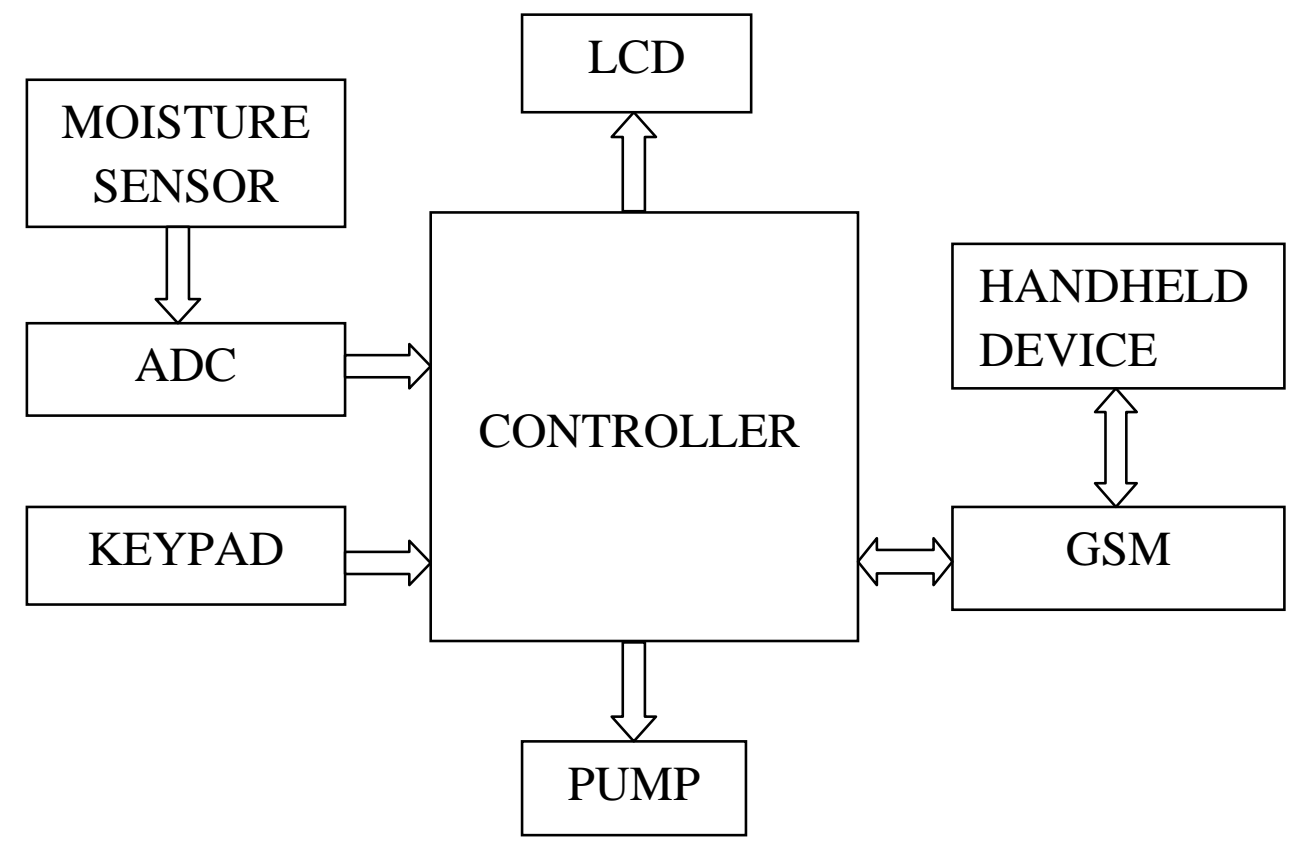

Figure 1: Block diagram of GSM based intelligent irrigation system

\subsection{Circuit function}

The intelligent irrigation system is based on microcontroller, which handle all the timing and switching functions. The microcontroller is used for processing the AT commands related to GSM. The MAX232 is interfaced with the microcontroller for the serial communication. The intelligent irrigation system also comprises of a display unit that can be an LCD display that display the signal received from microcontroller. A keypad is coupled to microcontroller to input the desired field moisture and save it so that the value can be compared with the value obtained from the field and accordingly the pump can be started or stopped. Another most important part of this system is the moisture sensor. It is capacitive moisture sensor that is used to obtain the moisture content from the field. The moisture content is directly proportional to the voltage output. The units in which the moisture content is measured are \%MC. The design details of the developed sensor are given in Table 1 .

Table 1. Design details of sensor

\begin{tabular}{|c|c|}
\hline Design parameters & Units \\
\hline Supply voltage & $5 \mathrm{v}-20 \mathrm{v}$ \\
\hline $\begin{array}{c}\text { Operational } \\
\text { temperature }\end{array}$ & $-20^{\circ} \mathrm{C}$ to $70^{\circ} \mathrm{C}$ \\
\hline $\begin{array}{c}\text { Power consumption } \\
\text { Power on to output } \\
\text { stable }\end{array}$ & $0.26 \mathrm{~mW}$ \\
\hline Output & 0 to $4.7 \mathrm{v}$ depending upon the \\
moisture content \\
\hline Surface area(tip)
\end{tabular}

\section{Field testing results}

The developed prototype was installed in the field at CDAC to test system reliability and performance. A test bed is created to check the performance and reliability of system. The details of test bed are given in Table 2 .

Table 2. Details of test bed

\begin{tabular}{|c|c|}
\hline Parameters & Units \\
\hline Temperature & $-20^{\circ} \mathrm{C}$ to $35^{\circ} \mathrm{C}$ \\
\hline Sensor depth & $30 \mathrm{~cm}$ \\
\hline Water source & Tube well reservoir \\
\hline Crop spacing & $15-20 \mathrm{~cm}$ \\
\hline $\begin{array}{c}\text { Irrigation time per } \\
\text { operation }\end{array}$ & 15 min \\
\hline $\begin{array}{c}\text { Irrigation interval } \\
\text { content }\end{array}$ & $\begin{array}{c}\text { Depending upon chosen moisture } \\
\text { Number of hours }\end{array}$ \\
\hline $\begin{array}{c}\text { Readings taken at } \\
\text { intervals }\end{array}$ & 4 \\
\hline
\end{tabular}

Using the test bed different readings are taken for the change in moisture content. The change in moisture content with respect to the change in temperature is shown in Table 3 . The graphical view of the change in moisture content with respect to temperature is shown in Figure 2. 
Table 3. Effect of temperature on moisture content

\begin{tabular}{|c|c|}
\hline Temperature $\left({ }^{\circ} \mathrm{C}\right)$ & Moisture content(\%MC) \\
\hline 22 & 44 \\
\hline 26 & 44 \\
\hline 32 & 41 \\
\hline 30 & 40 \\
\hline
\end{tabular}

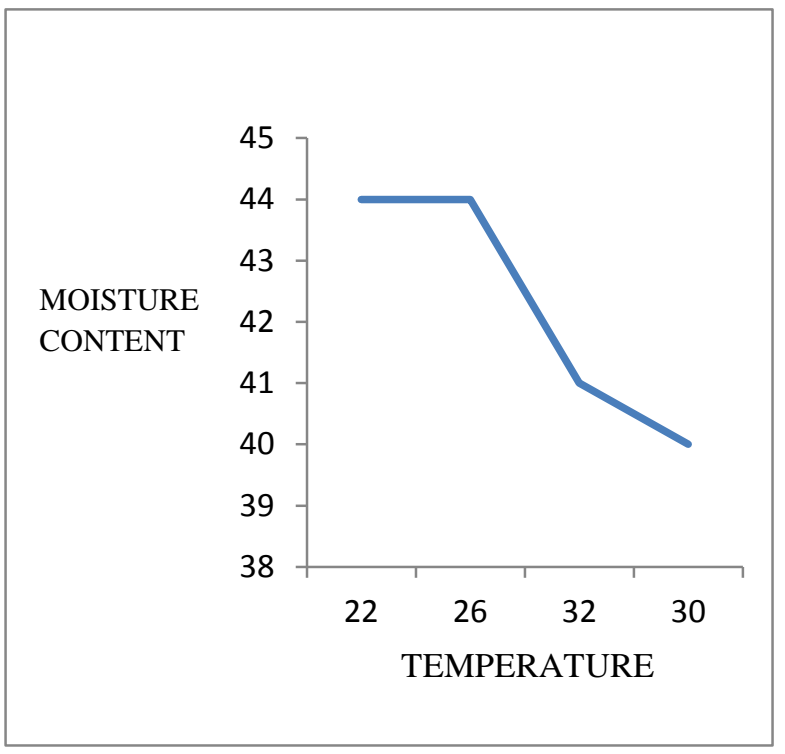

Figure 2: Graphical view of change in moisture content with respect to temperature

As seen from above graphical view we can see that the moisture content changes with the change in temperature. As the atmospheric temperature changes the values of moisture content changes. Now there is another factor with which the values of moisture content changes i:e time. The readings of moisture content with respect to time are given in Table 4 . In this we can see that as the time increases the moisture content decreases. The time is taken in hours. The graphical view of change in moisture content with respect to time is shown in Figure 3 . The readings are taken at four intervals.

\section{Table 4. Effect of time on moisture content}

\begin{tabular}{|c|c|}
\hline Time(hours) & Moisture content(\% MC) \\
\hline $08: 00$ & 44 \\
\hline $12: 00$ & 44 \\
\hline $16: 00$ & 42 \\
\hline $20: 00$ & 40 \\
\hline
\end{tabular}

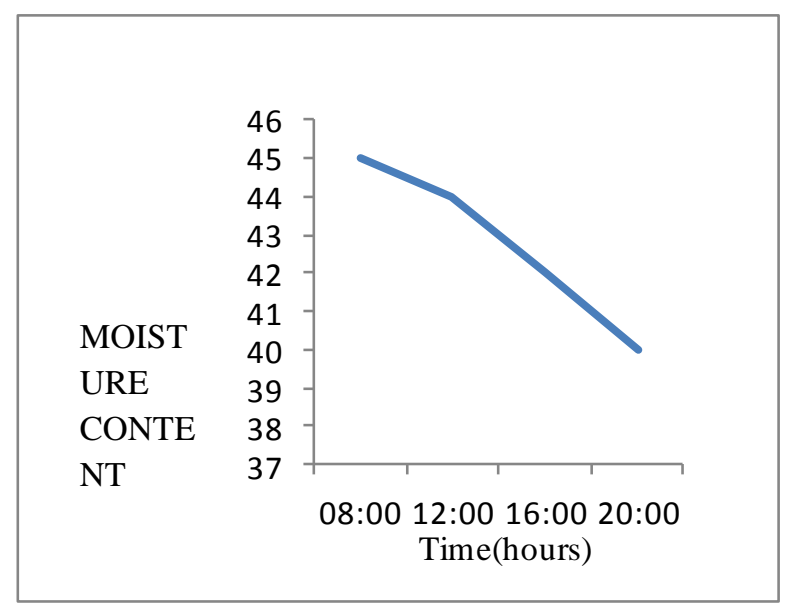

Figure 3: Graphical view of change in moisture content with respect to time

As seen from the above graphical view in Figure 3 the change in moisture is detected with change in time from morning to evening. Figure 4 shows the system testing in morning at 08:00 hours, temperature was $22^{\circ} \mathrm{C}$. Figure 5 shows the system testing in evening after irrigation at 16:00 hours, temperature was $32^{\circ} \mathrm{C}$.

Figure 4: System testing

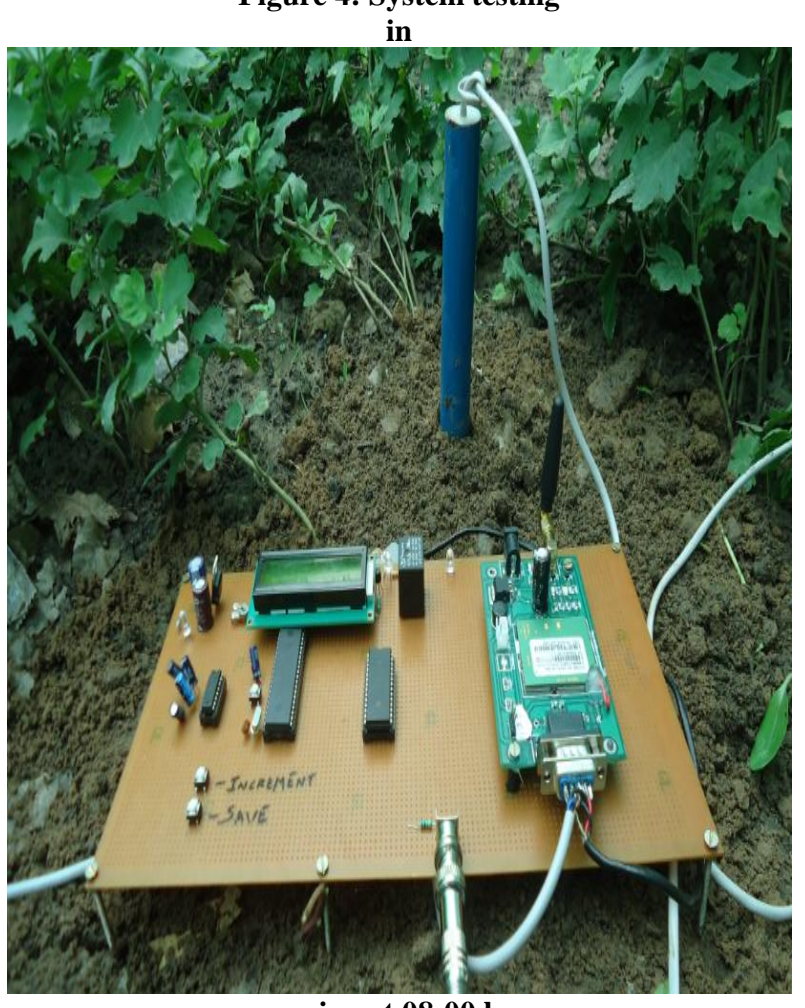

morning at 08:00 hours 


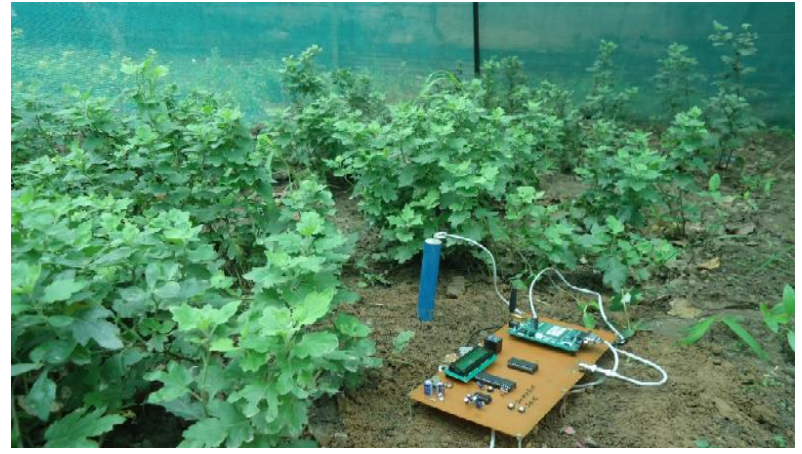

Figure 5: System testing in evening at 16:00 hours after irrigation

\section{CONCLUSION}

The indigenous intelligent developed system is more easy on the pocket and comprehensible for farmers. It consists of features and can be programmed according to the need of user. The moisture in the field is calculated up to the depth of $30 \mathrm{~cm}$ at various temperatures for the whole of 12 hours. In the at hand experiment the desired value of the moisture was set for the field in the morning and due to the effect of atmospheric temperature and the number of hours, the irrigation started when the moisture value gets lower than the desired value that was set. The irrigation time is 15 minutes so that the over irrigation can also be stopped to keep safe the fertilization. The readings of the moisture were taken remotely during the whole day. The developed intelligent irrigation system was found to be extremely user friendly, low cost and can be used for control irrigation, continuous and remote monitoring of the moisture at different places where the crop grown in open fields, at green house and orchards.

\section{ACKNOWLEDGEMENT}

We would like to express special thanks to CDAC (Centre for Development of Advanced Computing), Mohali for providing us all the facilities to carry our work properly and easily.
We would also like to thank MHRD, Government of India to support our work.

\section{REFERENCES}

[1] Adamo, F.; Andria, G.; Attivissimo, F.; Fabbiano, L.; Giaquinto, N.;, "Design and Development of a sensor prototype for Soil Moisture Measurement: First Experimental Results,"Instrumentation and Measurement Technology Conference Proceedings, 2007. IMTC 2007. IEEE, vol.2, no., pp.1-5, 1-3 May 2007.

[2] Yamamoto, Y.; Ogawa, A.;, "Novel soil-moisturesensors applying thermal characteristics of soil and water," Sensors, 2002. Proceedings of IEEE, vol.1, no., pp. 484- 489 vol.1, 2002.

[3] Xing Zhen; Shen Changjun; Zheng Wengang; Sun Gang; Zhou Jianjun; , "Research on the soil moisture sensor of three-probe structure, "World Automation Congress (WAC), 2010 , vol.3, no., pp.453-457, 19-23 Sept. 2010

[4] Rangan, K.; Vigneswaran, T., "An Embedded systems approach to monitor green house," Recent Advances in Space Technology Services and Climate Change (RSTSCC), 2010, vol.3, no., pp.61-65, 13-15 Nov. 2010.

[5] Zhuanwei Wang; Chunjiang Zhao; Haihui Zhang; Hongpan Fan, "Real-Time Remote Monitoring and Warning System in General Agriculture Environment," Information Technology, Computer Engineering and Management Sciences (ICM), 2011 International Conference, vol.3, no., pp.160-163, 24-25 Sept. 2011

[6] AT89s52 Datasheet, available at http://www.keil.com /dd/ docs/datashts/atmel/at89s52_ds.pdf.

[7] ADCO8O8 Datasheet, available at http://www.ti.com /lit/ds/symlink/adc0808-n.pdf. 